\title{
Universal Formats for Nonlinear Dynamical Systems
}

Krzysztof Kowalski

Department of Theoretical Physics, University of Eódź, ul. Pomorska 149/153, 90-236 Łódź, Poland

\begin{abstract}
It is demonstrated that very general nonlinear dynamical systems covering all cases arising in practice can be brought down to rate equations of chemical kinetics
\end{abstract}
Key words: nonlinear dynamical systems, chemical kinetics, chemical rate equa- tions, graphs of chemical reactions

PACS numbers: $\quad 02.10,02.90,82.20,82.30$ 
In recent years there has been great progress in the theory of classical dynamical systems of the form

$$
\dot{x}_{i}=F_{i}\left(x_{1}, \ldots, x_{k}\right), \quad i=1, \ldots, k
$$

where overdot denotes differentiation with respect to the time. Let us only recall the spectacular discovery of dynamical systems showing chaotic behaviour [1]. On the other hand, this part of the theory which deals with the universal properties of systems (1) can hardly be called satisfactory one. For example the Sixteenth Hilbert Problem (the problem of systematically counting and locating the limit cycle of polynomial systems on the plane) posed in 1900 remains unsolved even for systems with quadratic nonlinearity [2].

In order to study universal properties of dynamical systems (1) one should first find some universal object connected with (1). An attempt in this direction was made by Kerner [3] who showed that very general dynamical systems (1) embracing everything that arises in practice can be brought down to polynomial systems

$$
\dot{x}_{i}=P_{i}\left(x_{1}, \ldots, x_{N}\right), \quad i=1, \ldots, N,
$$

where $P_{i}$ are polynomials in $x_{1}, \ldots, x_{N}$, by introducing suitable new variables into the original system (1). The aim of this letter is to demonstrate that the polynomial systems (2) resulting from the original nonlinear dynamical systems (1) can be furthermore reduced to systems of rate equations of chemical kinetics by introducing appropriate additional variables. Thus we show in the present paper that very general dynamical systems (1) are represented by graphs of the corresponding chemical reactions.

We first recall the algorithm for construction of chemical rate equations from a given reaction mechanism. Let the species of the chemical reaction mechanism be $A_{1}, \ldots, A_{N}$. Elementary reactions are given by the stoichiometric equations 


$$
\sum_{i=1}^{N} m_{r i} A_{i} \stackrel{k_{r}}{\longrightarrow} \sum_{i=1}^{N} n_{r i} A_{i}, \quad r=1, \ldots, M,
$$

where $m_{r i}, n_{r i}$ are the stoichiometric coefficients, $m_{r i}, n_{r i} \in \mathbf{Z}_{+}$(the set of nonnegative integers) and $k_{r}$ is the rate constant; the formal sums from (3) are usually called complexes. According to the mass action law, the concentrations $x_{i}$ of the species $A_{i}, i=1, \ldots, N$ satisfy the following polynomial system

$$
\dot{x}_{i}=\sum_{r=1}^{M}\left(n_{r i}-m_{r i}\right) k_{r} \prod_{j=1}^{N} x_{j}^{m_{r j}}, \quad i=1, \ldots, N .
$$

Consider now the general polynomial system (2). One finds easily that it can be written in the form

$$
\dot{x}_{i}=\sum_{r=1}^{I_{i}} \alpha_{i r} \prod_{j=1}^{N} x_{j}^{n_{i r j}}-\sum_{s=1}^{J_{i}} \beta_{i s} \prod_{j=1}^{N} x_{j}^{m_{i s j}}, \quad i=1, \ldots, N
$$

where $\alpha_{i r}, \beta_{i s} \in \mathbf{R}_{+}$(the set of positive real numbers), the vectors $\mathbf{n}_{i r}, \mathbf{m}_{i s} \in \mathbf{Z}_{+}^{N}$ with coordinates $n_{i r j}$ and $m_{i s j}$, respectively are pairwise different and $I_{p}, J_{q} \in \mathbf{Z}_{+}$(it is understood that if $I_{p}$ or $J_{q}=0$ then the corresponding sum vanishes). The criterion for the polynomial system (5) to have an underlying chemical reaction mechanism has been found by Hárs and Tóth [4]. Namely, they observed that whenever the condition

$$
m_{i s i}>0 \quad \text { if } \quad J_{i}>0, \quad s=1, \ldots, J_{i}
$$

holds for arbitrary $i=1, \ldots, N$, then (5) is the system of rate equations corresponding to the following sequence of chemical reactions

$$
\begin{array}{r}
\sum_{j=1}^{N} n_{i r j} A_{j} \stackrel{\alpha_{i r}}{\longrightarrow} \sum_{j=1}^{N} n_{i r j} A_{j}+A_{i} \\
\sum_{j=1}^{N} m_{i s j} A_{j} \stackrel{\beta_{i s}}{\longrightarrow} \sum_{j=1}^{N} m_{i s j} A_{j}-A_{i} .
\end{array}
$$


It can be easily checked that the general system of rate equations (4) fulfils (6). Therefore, (6) is the necessary condition as well. It should also be noted that there can exist mechanisms different from (7) having the same kinetic rate equations (5). Nevertheless, the advantage of (7) is that it can be constructed quickly and algorithmically.

We now demonstrate that the general polynomial system (5) can be easily reduced to this one satisfying the condition (6) by introducing suitable new coordinates. Consider the system (5). Suppose that the condition (6) is not valid for $i=i_{1}, \ldots, i_{q} \in\{1, \ldots, N\}$. On introducing the new variables of the form

$$
x_{N+p}=1 / x_{i_{p}}, \quad p=1, \ldots, q
$$

we arrive at the following $N+q$-dimensional system satisfying the condition (6)

$$
\begin{aligned}
& \dot{x}_{i}=\sum_{r=1}^{I_{i}} \alpha_{i r} \prod_{j=1}^{N} x_{j}^{n_{i r j}}-\sum_{s=1}^{J_{i}} \beta_{i s} \prod_{j=1}^{N} x_{j}^{m_{i s j}}, \quad i \in\{1, \ldots, N\} \backslash\left\{i_{1}, \ldots, i_{q}\right\}, \\
& \dot{x}_{i_{p}}=\sum_{k=1}^{I_{i_{p}}} \alpha_{i_{p} k} \prod_{j=1}^{N} x_{j}^{n_{i_{p} k j}}-x_{i_{p}} x_{N+p} \sum_{l=1}^{J_{i_{p}}} \beta_{i_{p} l} \prod_{j=1}^{N} x_{j}^{m_{i_{p} l j}}, \\
& \dot{x}_{N+p}=-x_{N+p}^{2}\left(\sum_{k=1}^{I_{i_{p}}} \alpha_{i_{p} k} \prod_{j=1}^{N} x_{j}^{n_{i_{p} k j}}-\sum_{l=1}^{J_{i_{p}}} \beta_{i_{p} l} \prod_{j=1}^{N} x_{j}^{m_{i_{p} l j}}\right), \quad p=1, \ldots, q .
\end{aligned}
$$

We have thus shown that the general polynomial systems (5) can be brought down to these ones satisfying the condition (6) and the general dynamical systems (1) can be reduced to rate equations of chemical kinetics. An alternative method for the reduction of polynomial systems (2) to the chemical format has been recently reported by Samardzija et al [5]. Nevertheless, this method seems to be more complicated. The system (9) can be furthermore reduced to quadratic one by successive grouping $x_{j} x_{k}, j, k=1, \ldots, N+q$ as a single new variable [3]. Such reduction of polynomial systems (5) to quadratic rate equations is the ultimate one. Indeed, if one persists in grouping $x_{j} x_{k}$ as new variables then the linear infinite dimensional system is obtained (the nonlinear system (9) is embedded into infinite linear system 
- such embedding is called the Carleman one [6]). The possibility of asymptotical reduction of general polynomial systems (2) to quadratic rate equations describing conservative chemical reactions without autocatalytic steps was reported by Korzukhin [7]. Besides the perturbative nature the flaw of Korzukhin's reduction is its complexity. For example such simple equation as $\dot{x}=x$ is reduced (asymptotically) within the Korzukhin approach to rate equations corresponding to chemical reactions of the above mentioned type by introducing five new variables.

Example. Consider the Lorenz system

$$
\begin{aligned}
& \dot{x}_{1}=\sigma x_{2}-\sigma x_{1}, \\
& \dot{x}_{2}=r x_{1}-x_{2}-x_{1} x_{3}, \\
& \dot{x}_{3}=x_{1} x_{2}-b x_{3} .
\end{aligned}
$$

Owing to the term $-x_{1} x_{3}$ the system (10) is not "chemical" one. Taking into account (8) we introduce a new variable such that

$$
x_{4}=1 / x_{2} \text {. }
$$

The system (10) implies then the following system of rate equations

$$
\begin{aligned}
& \dot{x}_{1}=\sigma x_{2}-\sigma x_{1}, \\
& \dot{x}_{2}=r x_{1}-x_{2}-x_{1} x_{2} x_{3} x_{4}, \\
& \dot{x}_{3}=x_{1} x_{2}-b x_{3}, \\
& \dot{x}_{4}=x_{4}+x_{1} x_{3} x_{4}^{2}-r x_{1} x_{4}^{2} .
\end{aligned}
$$

The sequence of chemical reactions (7) corresponding to (11) is of the form 


$$
\begin{array}{cll}
A_{1}+A_{2}+A_{3} & & A_{1}+A_{2}+A_{3}+A_{4} \stackrel{1}{\rightarrow} A_{1}+A_{3}+A_{4} \\
\uparrow_{1} & A_{4} \stackrel{1}{\rightarrow} 2 A_{4} \\
A_{1}+A_{2} \stackrel{r}{\leftarrow} A_{1} & A_{1}+A_{3}+2 A_{4} \stackrel{1}{\rightarrow} A_{1}+A_{3}+3 A_{4} \\
\uparrow_{\sigma} \stackrel{\downarrow}{\downarrow} & A_{1}+2 A_{4} \stackrel{r}{\rightarrow} A_{1}+A_{4} \\
A_{2} \stackrel{1}{\longrightarrow} 0 \stackrel{b}{\leftarrow} A_{3} . &
\end{array}
$$

Here, 0 designates the zeroth complex and the reaction $A \rightarrow 0(0 \rightarrow A)$ means that the substance A is removed from (supplied to) the chemical reactor. In order to reduce (11) to the system of quadratic rate equations we now introduce the following new variables

$$
x_{5}=x_{1} x_{4}, \quad x_{6}=x_{3} x_{5} .
$$

Making use of (11) one finds easily that in this variables the vector fields $\dot{x}_{i}$, where $i \neq 4$ do not depend on $x_{4}$ and therefore the variable $x_{4}$ can be omitted from the six-dimensional system obtained via (11) and (12). On renumbering $x_{6} \rightarrow x_{4}$ we finally arrive at the system of quadratic rate equations such that

$$
\begin{aligned}
& \dot{x}_{1}=\sigma x_{2}-\sigma x_{1}, \\
& \dot{x}_{2}=r x_{1}-x_{2}-x_{2} x_{4}, \\
& \dot{x}_{3}=x_{1} x_{2}-b x_{3}, \\
& \dot{x}_{4}=\sigma x_{3}+x_{1}^{2}+x_{4}^{2}-(\sigma+b-1) x_{4}-r x_{4} x_{5}, \\
& \dot{x}_{5}=\sigma+x_{4} x_{5}-(\sigma-1) x_{5}-r x_{5}^{2},
\end{aligned}
$$

where $x_{4}=x_{1} x_{3} / x_{2}, x_{5}=x_{1} / x_{2}$. The corresponding chemical reaction mechanism (7) can be written as 


$$
\begin{aligned}
& A_{4}+2 A_{5} \quad 2 A_{5} \\
& 1 \uparrow \quad r \downarrow \\
& A_{4}+A_{5} \stackrel{r}{\rightarrow} A_{5} \\
& \sigma-1 \downarrow \uparrow \sigma \\
& A_{2}+A_{4} \stackrel{1}{\rightarrow} A_{4} \stackrel{\sigma+b-1}{\longrightarrow} 0 \stackrel{b}{\leftarrow} A_{3} \stackrel{\sigma}{\rightarrow} A_{3}+A_{4} \\
& \sigma \nearrow \nwarrow 1 \\
& A_{1} \quad A_{2} \\
& r \searrow \swarrow \sigma \\
& A_{1}+A_{2}+A_{3} \stackrel{1}{\leftarrow} A_{1}+A_{2} \quad 2 A_{4} \stackrel{1}{\rightarrow} 3 A_{4} .
\end{aligned}
$$

In summary, we have demonstrated in this work that very general classical dynamical systems can be reduced to rate equations of chemical kinetics. The cost of such reduction is an increase in dimension of systems resulting from introducing new variables and restrictions imposed on initial data by the requirement of nonnegativity of concentrations. In spite of these limitations, the point remains that general dynamical systems can be viewed as kinetic rate systems and thus they are represented by graphs of the corresponding chemical reactions. The problem of possible applications of this result remains open. On the one hand, working in the field of nonlinear models will find that existing surprisingly strong theorems relating the dynamics of rate equations with the structure of the underlying graph of a chemical reaction mechanism such as zero defficiency theorem [8], the theorem on knots of trees [9] and Volpert theorems [10] (see also [11]) cannot be used in the case of graphs such as (13) corresponding to reaction mechanisms with autocatalytic steps. On the other hand, as it was mentioned by a referee, a chemist is most interested in inverse problem: "how to find a simple model underlying the complex reality of a typical chemical mechanism" (in the light of the observations presented herein it is really difficult job). Nevertheless, maybe at some distant stage in the future our 
experimental ability will be such that it would be possible to "design" particular chemical reactor that fit given scheme and, for example, one would predict behaviour of a complex mechanical system from the colour of a chemical mixture.

\section{Acknowledgement}

I would like to thank a referee for helpful comments. 


\section{References}

[1] E.N. Lorenz, J. Atmos. Sci. 20 (1972) 130.

[2] D.E. Koditschek and K.S. Narendra, J. Differential Equations 54 (1984) 181.

[3] E.H. Kerner, J. Math. Phys. 22 (1981) 1366.

[4] V. Hárs and J. Tóth, in: Qualitative Theory of Differential Equations, Szeged, 1979, Colloq. Math. Soc. János Bolyai 30.

[5] N. Samardzija, L.D. Geller and E. Wasserman, J. Chem. Phys. 90 (1989) 2296.

[6] K. Kowalski and W.-H. Steeb, Nonlinear Dynamical Systems and Carleman Linearization (World Scientific, Singapore, 1991).

[7] M.D. Korzukhin, in: Oscillatory Processes in Biological and Chemical Systems (Nauka, Moscow, 1967) (in Russian).

[8] M. Feinberg, in: Dynamics and Modelling of Reactive Systems (Academic Press, New York, 1980).

[9] B.L. Clarke, Adv. Chem. Phys. 43 (1980) 7.

[10] A.I. Volpert and S.I. Khudayev, Analysis in Classes of Discontinuous Functions and Equations of Mathematical Physics (Nauka, Moscow, 1975) (in Russian).

[11] A.N. Gorban, V.I. Bykov and G.S. Yablonski, Essays on Chemical Relaxation (Nauka, Novosibirsk, 1986) (in Russian). 